Original Research Article

\title{
Potentially inappropriate prescribing in elderly: a comparison of Beers and STOPP criteria in tertiary care
}

\author{
Akanksha Mathur*, Prashant C. Shah
}

Department of Pharmacology,

Baroda Medical College,

Vadodara, Gujarat, India

Received: 10 October 2018

Accepted: 01 November 2018

*Correspondence to:

Dr. Akanksha Mathur,

Email: akanksha.mathur1@ gmail.com

Copyright: (C) the author(s), publisher and licensee Medip Academy. This is an openaccess article distributed under the terms of the Creative Commons Attribution NonCommercial License, which permits unrestricted noncommercial use, distribution, and reproduction in any medium, provided the original work is properly cited.

\begin{abstract}
Background: Prescribing in elderly is a challenging task as they have age related physiological changes, various co-morbidities, altered pharmacological properties and higher propensity for adverse events. They are often prescribed medications which are potentially inappropriate for them, sometimes may even be unnecessary. The medicines are considered as inappropriate if the risk associated with them outweighs benefits. The objective of this study is to assess the prevalence of potentially inappropriate medications (PIM) at a tertiary care teaching hospital according to the Beers updated 2015 criteria and STOPP criteria and to compare the two criteria in detection of PIMs.
\end{abstract}

Methods: A prospective observational study involving 228 elderly patients (>65years) of medicine wards was conducted from October 2015 to March 2016. Relevant information was recorded in a predesigned proforma. The use of potentially inappropriate medications is assessed using Beers updated 2015 criteria and STOPP criteria using descriptive statistics.

Results: The prevalence of PIM use in the sample was $26.31 \%$ according to the 2015 Beers criteria and $14.03 \%$ using the STOPP criteria. The most prevalent PIM according to the Beers criteria were sliding scale insulin $(17.54 \%)$ and long acting benzodiazepines (5.26\%); according to the STOPP criteria, they were aspirin in heart failure $(5.26 \%)$ and chlorpheniramine $(3.07 \%)$.

Conclusions: The prevalence of PIM varied when different criteria were applied. The 2015 Beers criteria identified more PIM than the STOPP criteria.

Keywords: Beers criteria, Geriatrics, Potentially inappropriate medications, STOPP criteria

\section{INTRODUCTION}

Drug therapy in elderly is a challenging task as the elderly tend to have reduced physiological reserve, altered pharmacokinetics, multiple comorbidities thus leading to polypharmacy and drug-drug interactions. Moreover, they are more prone to develop adverse effects due to drugs. ${ }^{1,2}$ Certain medications are termed as potentially inappropriate for the use in elderly because the risk of adverse events outweighs the clinical benefits. This is particularly applicable when there is a better alternative available for the same condition. ${ }^{3,4}$ Prescription of these medications not only leads to adverse drug reactions, but increases the morbidity and mortality, and also wastes the scarce health resources. ${ }^{5}$

The prevalence of inappropriate medication use has been analyzed in several studies. It ranges from 20 to $79 \%$ depending on the type of population, the setting and the specific tool used. ${ }^{3}$

Several approaches have been made to detect potentially inappropriate medications. One of the most commonly employed is the Beers criteria. It was developed by a geriatrician, Mark H. Beers in 1991 and last updated in 2015 by the American Geriatrics Society. ${ }^{6}$ The prevalence of PIM identified by it ranges from 18 to $42 \%$ in several 
studies conducted in different countries. ${ }^{7}$ Another newer assessment tool, 'Screening Tool of Older Person's Prescriptions' (STOPP) has also been developed in 2008 with latest version published in $2015 .^{8}$ Various studies demonstrated that this newer criteria identified more number of PIM as compared to Beers criteria but the results are variable. ${ }^{7,9-11}$

This study was done to assess and characterize the prevalence of potentially inappropriate medications (PIM) at a tertiary care teaching hospital according to the Beers 2015 criteria and STOPP criteria and to compare the two criteria in detection of PIM.

\section{METHODS}

A prospective observational study was conducted in medicine wards at S.S.G. Hospital, Baroda for a duration of six months extending from October 2015 to March 2016. The study protocol was approved by Institutional Ethics Committee prior to the commencement of the study.

Total 228 elderly inpatients from the medicine department were included in the study. The sample size was decided on the basis of pilot study. Patients of either sex of age 65 years or more were included in the study. Patients who were unwilling to participate in the study were excluded from the study. All the patients who participated in the study were given clear explanations about the purpose and nature of the study in the language they understood. Written informed consent was taken from every patient who participated in the study.

The study was carried out by regular visits to medicine wards and case sheets from elderly inpatients were collected. The relevant data collected from case sheets were properly documented in a separate data collection form.

\section{Following parameters were assessed}

- Gender distribution

- Age wise distribution

- Average duration of hospital stays

- Organ system wise/ etiology wise distribution

- Pattern of drugs prescribed

- Average number of drugs prescribed per admission

- Total number of potentially inappropriate medications (PIM) using Beers criteria 2015 and STOPP criteria. $^{12,13}$

- Pattern of PIM prescription

- Comparisons between the 2 sets of criteria were made by noting the frequency with which certain drugs were identified as potentially inappropriate.

\section{Statistical analysis}

The data obtained was recorded in Microsoft Excel spreadsheet. Descriptive statistics were used to evaluate the data in terms of frequencies, percentage, range and mean \pm SD along with appropriate graphical displays.

\section{RESULTS}

A total of 228 cases were analyzed and out of which 147 $(64.47 \%)$ were females and $81(35.52 \%)$ were males. The average age of patients was $70.90 \pm 6.25$ years, the overall range being 65-97years. Average hospital stay was 4.82 days $(95 \% \mathrm{CI}=4.47-4.74)$.

Central nervous system (32.78\%) was the most commonly involved followed by cardiovascular system (25.49\%) and respiratory system $(8.94 \%)$ (Figure 1).

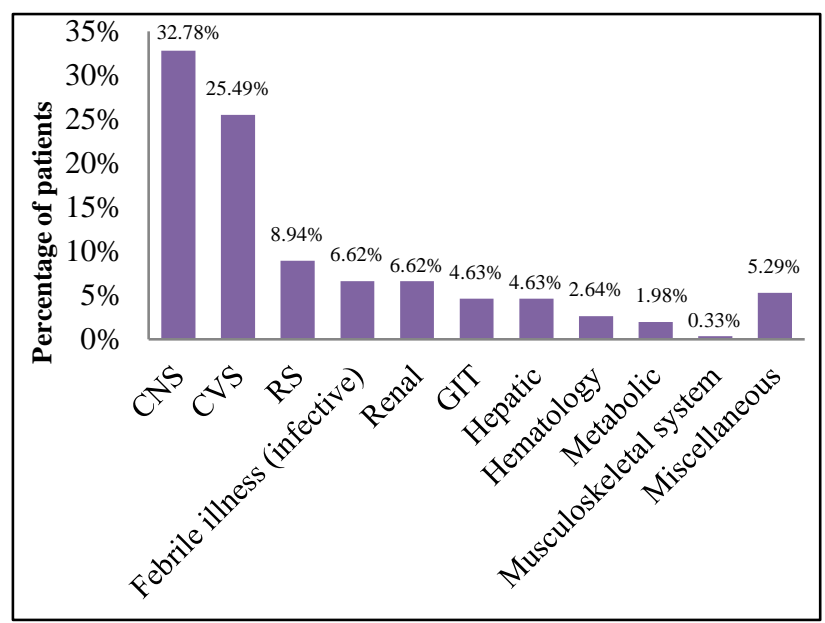

Figure 1: Etiology/ organ system wise distribution.

The number of medications prescribed ranged from 1 to 20 . Average number of drugs prescribed was $9.37 \pm 0.35$ with 80 patients $(35.08 \%)$ being prescribed 10 or more medications (Table 1). In total, 2295 drugs were prescribed to all the patients, and 14 different medications were identified as PIMs according to either the Beers or STOPP criteria.

Table 1: Prescribing frequency of drugs.

\begin{tabular}{|ll|}
\hline No. of drugs & Prescribing frequency $\mathbf{n}(\%)$ \\
\hline$\leq 5$ & $15(6.57 \%)$ \\
\hline $6-10$ & $118(51.75 \%)$ \\
\hline $11-15$ & $80(35.08 \%)$ \\
\hline$>16$ & $15(6.57 \%)$ \\
\hline
\end{tabular}

Without differentiating between Beers and STOPP criteria, the most common type of PIM prescribed was insulin as sliding scale in $40(17.54 \%)$ patients. The next most frequent PIM prescribed was a long acting benzodiazepine for insomnia in $13(5.70 \%)$ patients followed by aspirin in heart failure patients $12(5.26 \%)$.

According to Beers criteria, 84 (3.66\%) medicines were potentially inappropriate 2015 (Table 2). These were distributed amongst $26.31 \%(n=60)$ of the study 
population with 51 patients $(22.36 \%)$ receiving one PIM, 9 $(3.94 \%)$ receiving more than one PIM per prescription. The most common type of PIM prescribed was insulin as sliding scale in $40(17.54 \%)$ patients.

Long acting benzodiazepine (diazepam, clonazepam) for insomnia in $13(5.70 \%)$ patients and spironolactone $>25 \mathrm{mg} /$ day in $8(3.50 \%)$ patients were other PIMs detected by Beers criteria.

Table 2: PIM according to Beers criteria 2015.

\begin{tabular}{|ll|}
\hline Drug & $\begin{array}{l}\text { Number of } \\
\text { patients [n (\%)] }\end{array}$ \\
\hline $\begin{array}{l}\text { Digoxin prescribed in dose } \\
>0.125 \mathrm{mg} / \text { day }\end{array}$ & $6(2.63 \%)$ \\
\hline Insulin as sliding scale & $40(17.54 \%)$ \\
\hline $\begin{array}{l}\text { Long acting benzodiazepine } \\
\text { (diazepam, clonazepam) for } \\
\text { insomnia }\end{array}$ & $13(5.70 \%)$ \\
\hline $\begin{array}{l}\text { Intermediate acting } \\
\text { benzodiazepine (lorazepam) }\end{array}$ & $2(0.87 \%)$ \\
\hline Spironolactone $>25 \mathrm{mg} /$ day & $8(3.50 \%)$ \\
\hline Prazosin as antihypertensive & $2(0.87 \%)$ \\
\hline Clonidine as hypertensive & $2(0.87 \%)$ \\
\hline Nifedipine, immediate release & $1(0.43 \%)$ \\
\hline Chlorpheniramine & $7(3.07 \%)$ \\
\hline $\begin{array}{l}\text { Amiodarone as first line therapy } \\
\text { for atrial fibrillation }\end{array}$ & $1(0.43 \%)$ \\
\hline Trihexyphenidyl & $1(0.43 \%)$ \\
\hline $\begin{array}{l}\text { Digoxin as the first line for atrial } \\
\text { fibrillation }\end{array}$ & $1(0.43 \%)$ \\
\hline
\end{tabular}

Table 3: PIM according to STOPP criteria.

\begin{tabular}{|ll|}
\hline Drug & $\begin{array}{l}\text { Number of } \\
\text { patients [n (\%)] }\end{array}$ \\
\hline Chlorpheniramine for fallers & $7(3.07 \%)$ \\
\hline Aspirin in heart failure & $12(5.26 \%)$ \\
\hline Digoxin in dose >125mcg/day & $5(2.19 \%)$ \\
\hline Warfarin with NSAID & $3(1.31 \%)$ \\
\hline Diazepam for fallers & $5(2.19 \%)$ \\
\hline $\begin{array}{l}\text { Prazosin in patient of frequent } \\
\text { incontinence }\end{array}$ & $1(0.43 \%)$ \\
\hline $\begin{array}{l}\text { Aspirin with warfarin without H2 } \\
\text { blocker }\end{array}$ & $1(0.43 \%)$ \\
\hline
\end{tabular}

When STOPP criteria were applied, 34 (1.48\%) medications were found to be potentially inappropriate (Table 3).

Total $32(14.03 \%)$ patients received PIM according to STOPP criteria, out of which $30(13.15 \%)$ patients received one PIM while $2(0.87 \%)$ were prescribed more than two PIM per prescription. Aspirin in heart failure patients 12 (5.26\%) was the most common PIM identified followed by chlorpheniramine for fallers in $7(3.07 \%)$ patients and digoxin in dose $>125 \mathrm{mcg} /$ day in $5(2.19 \%)$ patients.

\section{DISCUSSION}

The study showed high average number of drugs being prescribed to the patients. This is also observed in other studies. ${ }^{14-16}$ This can be due to multiple co-morbid conditions in elderly, leading to a higher number of drugs being prescribed per patient. Although polypharmacy increases the risk of adverse effects, drug-drug interactions and increased cost of treatment, it becomes unavoidable in many patients.

Majority of the patients had involvement of central nervous system followed by cardiovascular and respiratory system. The pattern of diseases prevalent in elderly was similar to other studies. ${ }^{17-19}$

The common PIM identified by both the criteria, Beers 2015 as well as STOPP criteria were first generation antihistamines, long acting benzodiazepines and alpha blockers. ${ }^{4,9,20}$ First generation antihistamines are highly anticholinergic, so risk of confusion, dry mouth, constipation, and other anticholinergic effects increases; its clearance also reduces with advanced age. ${ }^{12}$ Long acting benzodiazepines causes prolonged sedation and increased incidence of falls and fractures. ${ }^{21}$ Alpha blockers have a high risk of orthostatic hypotension.

According to the Beers criteria, use of sliding scale insulin in elderly should be avoided. That was the most commonly observed PIM by Beers criteria. Also, it was found that digoxin was prescribed in dose $>0.125 \mathrm{mg} /$ day for heart failure or atrial fibrillation. It is considered as inappropriate for elderly as its clearance decreases with age. ${ }^{22}$

The prevalence of potentially inappropriate medications being prescribed is found to be variable when two different assessment tools were applied (Beers 2015 criteria and STOPP criteria). It was $26 \%$ by Beers 2015 criteria while $14 \%$ using STOPP criteria. The PIM prevalence assessed by Beers criteria is similar to other studies. ${ }^{6,9,23}$

In present study, Beers criteria identified more PIM than STOPP. This finding is also observed in studies conducted at Brazil and India while several other studies showed increased PIMs detection by STOPP criteria as compared to Beers criteria. ${ }^{9-11,13,23}$ This can be due to the use of older versions of Beers criteria in these studies. Earlier versions of Beers criteria had a simple list of inappropriate medications, no categorization of PIMs and inclusion of several older medications that were no longer available. ${ }^{24}$ Moreover, the criteria in STOPP assessment tool are more related to detection of PIM in a specific condition or a disease setting. Present study finding of increased PIM detection could be due to listing of drugs as potentially inappropriate independent of diagnosis/ condition by Beers criteria.

However, the study has certain limitations. The study was conducted at a single hospital in a developing country thus the findings cannot be generalized. Moreover, it included 
only inpatients of medicine wards, thus introducing selection bias.

Nevertheless, it detected the potentially inappropriate medications being prescribed to elderly which can be avoided. The results of this study may help in creating awareness among health care professionals regarding prescription in elderly. Further studies may involve larger sample size, investigate justification for the use of PIM and may involve association of risk factors with potentially inappropriate medications.

Funding: No funding sources

Conflict of interest: None declared

Ethical approval: The study was approved by the Institutional Ethics Committee

\section{REFERENCES}

1. Sandhiya S, Adithan C. Drug Therapy in the Elderly. J Assoc Physicians India. 2008;56:525-31.

2. Noble RE. Drug therapy in the elderly. Metabolism. 2003;52(10 Suppl 2):27-30.

3. Renom-Guiteras A, Meyer G, Thürmann PA. The EU (7)-PIM list: a list of potentially inappropriate medications for older people consented by experts from seven European countries. Eur J Clin Pharmacol. 2015 Jul 1;71(7):861-75.

4. Osei EK, Berry-Cabán CS, Haley CL, Rhodes-Pope H. Prevalence of Beers criteria medications among elderly patients in a military hospital. Gerontol Geriatric Med. 2016 Mar 12;2:2333721416637790.

5. de Lima TJV. Garbin CAS. Garbin AJI, Sumida DH. Saliba O. Potentially inappropriate medications used by the elderly: prevalence and risk factors in Brazilian care homes. BMC Geriat. 2013;13:52.

6. Shah KN, Joshi HM, Christian RP, Patel KP, Malhotra SD. Prevalence of potentially inappropriate medications and prescription cost analysis among older cardiac patients in an outpatient department of a tertiary care hospital in India. J Basic Clin Pharmacy. 2016 Sep;7(4):110-5.

7. Chang CB, Chen JH, Wen CJ, Kuo HK, Lu IS, Chiu LS, et al. Potentially inappropriate medications in geriatric outpatients with polypharmacy: application of six sets of published explicit criteria. Br J Clin Pharmacol. 2011 Sep;72(3):482-9.

8. Dalleur O, Boland B, De Groot A, Vaes B, Boeckxstaens P, Azermai M, et al. Detection of potentially inappropriate prescribing in the very old: cross-sectional analysis of the data from the BELFRAIL observational cohort study. BMC Geriatrics. 2015 Dec;15(1):156.

9. Gallagher P, O’Mahony D. STOPP (Screening Tool of Older Persons' potentially inappropriate Prescriptions): application to acutely ill elderly patients and comparison with Beers' criteria. Age Ageing. 2008 Oct 1;37(6):673-9.

10. Ryan C, O'mahony D, Kennedy J, Weedle P, Byrne S. Potentially inappropriate prescribing in an Irish elderly population in primary care. $\mathrm{Br} \mathrm{J}$ Clin Pharmacol. 2009 Dec 1;68(6):936-47.

11. Oliveira MG, Amorim WW, de Jesus SR, Heine JM, Coqueiro HL, Passos LC. A comparison of the Beers and STOPP criteria for identifying the use of potentially inappropriate medications among elderly patients in primary care. J Evaluation Clin Practice. 2015 Apr;21(2):320-5.

12. The American Geriatrics Society. American geriatrics society 2015 updated beers criteria for potentially inappropriate medication use in older adults. J Am Geriatr Soc. 2015 Nov;63(11):2227-46.

13. O'mahony D, O'sullivan D, Byrne S, O'connor MN, Ryan C, Gallagher P. STOPP/START criteria for potentially inappropriate prescribing in older people: version 2. Age Ageing. 2015 Mar 1;44(2):213-8.

14. Goudanavar P, Keerthi Y, Jacob J, Krishna R. A Prospective Study on Medication Prescribing Pattern for Geriatric Patients in a Tertiary Care Teaching Hospital. Asian J Biomed Pharmaceut Sci. 2016 Jun 10;6(56).

15. Abraham F, Varughese G, Mathew JC, John PM, Sam GK. Drug utilization pattern among geriatric patients in a tertiary care teaching hospital. Asian J Pharm Clin Res. 2015;8(6):191-4.

16. Jhaveri BN, Patel TK, Barvaliya MJ, Tripathi CB. Drug utilization pattern and pharmacoeconomic analysis in geriatric medical in-patients of a tertiary care hospital of India. J Pharmacol Pharmacotherapeut. 2014 Jan;5(1):15-20.

17. Ramesh KT, Shahina S, Shobha JC, Naidu MU. Usha Rani, 192 Vijay T. Drug utilization in geriatric population in a tertiary care 193 center. JK science. 1999;3:118-20.

18. Shah RB, Gajjar BM, Desai SV. Drug utilization pattern among geriatric patients assessed with the anatomical therapeutic chemical classification/defined daily dose system in a rural tertiary care teaching hospital. Int J Nutr Pharmacol Neurological Dise. 2012 Sep 1;2(3):258-65.

19. Shankar PR, Upadhyay DK, Subish P, Bhandari RB, Das B. Drug utilisation among older inpatients in a teaching hospital in Western Nepal. Singapore Med J. 2010 Jan 1;51(1):28-34.

20. Sah AK, Jha RK, Sah P, Basnet S. Potentially inappropriate prescribing in elderly population: A study in medicine out-patient department. J Coll Med Sci-Nepal. 2017 Jan 1;13(1):197-202.

21. Van der Hooft CS, Schoofs MW, Ziere G, Hofman A, Pols HAP, Sturkenboom MC, et al. Inappropriate benzodiazepine use in older adults and the risk of fracture. Br J Clin Pharmacol. 2008 Aug;66(2):27682.

22. Currie GM, Wheat JM, Kiat H. Pharmacokinetic considerations for digoxin in older people. Open Cardiovas Med J. 2011;5:130-5.

23. Nagendra Vishwas H, Harugeri A, Parthasarathi G, Ramesh M. Potentially inappropriate medication use in Indian elderly: Comparison of Beers' criteria and screening tool of older persons' potentially 
inappropriate Prescriptions. Geriatrics Gerontol Int. 2012 Jul;12(3):506-14.

24. Lim YJ, Kim HY, Choi J, Lee JS, Ahn AL, Oh EJ, et al. Potentially inappropriate medications by beers criteria in older outpatients: prevalence and risk factors. Korean J Famy Med. 2016 Nov 1;37(6):32933.
Cite this article as: Mathur A, Shah PC. Potentially inappropriate prescribing in elderly: a comparison of Beers and STOPP criteria in tertiary care. Int J Basic Clin Pharmacol 2019;8:95-9. 